\title{
Enhancement of Bioavailability of Fenofibrate with Alpha Tocopherol and Phospholipids as Solubilizers
}

\author{
TK Indira ${ }^{1 *}$, PK Lakshmi ${ }^{1}$, J Balasubramaniam² and YV Rajesh ${ }^{2}$
}

${ }^{1}$ Department of Pharmaceutics, Osmania University, Hyderabad, Andhra Pradesh 500028, India

${ }^{2}$ Research and Development Centre, RA Chem Pharma Limited, Hyderabad, Andhra Pradesh 500076, India

\begin{abstract}
The purpose of this study was to investigate the effect of different solubilizers namely alpha tocopherol, soy phosphatidylcholine 70 , Phospholipon $80 \mathrm{H}$, and Phospholipon $90 \mathrm{H}$ on the bioavailability of sustained release fenofibrate pellets using fluid bed coating by applying Taguchi design to optimize the type and concentration of solubilizer at four levels namely $0.5 \%, 1 \%, 1.5 \%$, and $2 \%$. The pellets were prepared by loading the fenofibrate blended with other excipients onto the core sugar pellets with the aid of the binder solution. Taguchi experimental runs with alpha tocopherol $1 \%$ and Phospholipon $90 \mathrm{H} 2 \%$ (test) showed significant differences in in vitro dissolution behavior of drug compared to the pure drug. The pharmacokinetics of pure drug and test was evaluated in healthy male Wistar rats and found that $t_{1 / 2}$ was reduced significantly ( 4.36 and 4.02 hours) while $A U C_{0-t}(32.14 \pm 6.38 \mu \mathrm{g} \mathrm{h} / \mathrm{ml}, 36.94 \pm 6.2 \mu \mathrm{g}$ $\mathrm{h} / \mathrm{ml}), \mathrm{C}_{\max }(8.7 \pm 2.31 \mu \mathrm{g} / \mathrm{ml}, 9.8 \pm 2.2 \mu \mathrm{g} / \mathrm{ml})$ were improved markedly compared to the pure drug with $\mathrm{t}_{1 / 2}(7.339314$ \pm 3.1 hours $), A C_{0-1}(11.89 \pm 8.13 \mu \mathrm{g} \mathrm{h} / \mathrm{ml})$, and $C_{\text {max }}(5.137 \pm 3.37 \mu \mathrm{g} / \mathrm{ml})$. The extent of the mean plasma exposure of fenofibrate was 2.7 and 3.1 fold higher in animals treated with test. The ANOVA results revealed that type and concentration of solubilizer are crucial for enhancement of in vitro dissolution profile. Hence use of solubilizers may be the promising way to improve the oral bioavailability of fenofibrate.
\end{abstract}

\section{Introduction}

Oral drug delivery continued to be the preferred route of drug administration especially in instances of multiple frequency of dosing. However, the effective drug delivery through oral route still remains a major challenge for highly lipophilic drugs in the pharmaceutical field owing to their poor bioavailability [1]. Prerequisite for effective oral drug delivery is the dissolution of pharmaceutical drug formulation in the gastro-intestinal lumen. It has been estimated that about 40 to 70 percent of New Chemical Entities (NCE's) entering drug development process possess inadequate aqueous solubility resulting in poor absorption thereby resulting in poor bioavailability [2].

Several attempts had been made in literature to enhance the oral bioavailability of poorly soluble and highly lipophilic drugs. More recently, micronized fenofibrate showed significantly improved dissolution and enhanced oral bioavailability [3]. The present study aims at enhancement of oral bioavailability of fenofibrate. Fenofibrate is a lipid lowering highly lipophilic drug with low water solubility and high membrane permeability included in class II of biopharmaceutical drug classification system. It has a lipid-water distribution coefficient of 5.24 and aqueous solubility of less than $0.5 \mathrm{mg} /$ liter with half life of 20 hours. Due to its virtual aqueous insolubility it is poorly absorbed from digestive tract leading to consequences of incomplete, irregular oral bioavailability [4]. The therapeutic dose required to be administered must thus be increased to obviate the disadvantage.

To decrease the daily dose of drug and frequency of administration, fenofibrate was formulated into sustained release pellets by incorporating HPMC K4M as sustained release matrix forming polymer. The drug and excipient blend was loaded on to the core pellets with the aid of the binder solution and various process parameters were optimized using fluid bed coater that is more automated and advanced technique compared to conventional pan coating. The potential use of vitamin E preferably alpha tocopherol as antioxidant to protect plasma low density lipoproteins [5] and the use of alpha tocopherol as solubilizer for poorly soluble and highly lipophilic drug griseofulvin is well reported [6]. Owing to the dual advantages Alpha Tocopherol (AT) was selected as solubilizer for fenofibrate. Presence of food increased absorption of fenofibrate by nearly $35 \%$ compared with fasting conditions [7]. Therefore different phospholipids namely Soy Phosphatidyl Choline 70 (SPC 70), Phospho Lipon 80H (PL 80H) and Phospho Lipon 90H (PL 90H) were incorporated as solubilizers owing to advantages that they occur naturally in biological membranes, freely available in nature, relatively effective at low concentrations. Phospholipids are physiological lipids present in all living species. Among all phospholipids, phosphatidylcholine (PC) is the most abundant biomolecule and the only one available in pure form on industrial scale. Phosphatidylcholine is non-toxic and no limits have been established for maximum daily intake. However, their potential usage as oral drug delivery carriers for poorly water-soluble fenofibrate has not been investigated. In this study, fenofibrate-loaded alpha tocopherol and phospholipids sustained release pellets were prepared and evaluated both in vitro and in vivo.

\section{Materials and Methods}

\section{Materials}

Fenofibrate (unmicronized) was obtained from Alembic Private Limited (Gujarat, India), Povidone K30 (Plasdone K 29/32') was

*Corresponding author: TK Indira, Department of Pharmaceutics, Osmania University, Hyderabad, Andhra Pradesh, India, Tel: 09396555519; E-mail: indu.induroy@gmail.com

Received April 24, 2012; Accepted May 12, 2012; Published May 14, 2012

Citation: Indira TK, Lakshmi PK, Balasubramaniam J , Rajesh YV (2012) Enhancement of Bioavailability of Fenofibrate with Alpha Tocopherol and Phospholipids as Solubilizers. J Bioequiv Availab S14. doi:10.4172/jbb.S14-006

Copyright: ( 2012 Indira TK, et al. This is an open-access article distributed under the terms of the Creative Commons Attribution License, which permits unrestricted use, distribution, and reproduction in any medium, provided the original author and source are credited. 
obtained from ISP (New Jersey, USA), HPMC-E5 (Pharmacoat $606^{\circ}$ ) was obtained from SHIN-ETSU (Tokyo, Japan), HPMC K4M (Methocel K4M ${ }^{*}$ ) was obtained from Dow chemicals (Asia Pacific). Alpha tocopherol was obtained from (BASF, Norway), soy phosphatidyl choline 70 (containing phosphatidyl choline 70\%) was obtained from Sonic Biochem Extractions (Madhya Pradesh, India). Phospholipon $80 \mathrm{H}$ (containing $70 \%$ of phosphatidyl choline, $6 \%$ of lysophosphatidyl choline) and Phospholipon $90 \mathrm{H}$ (containing 90\% of phosphatidyl choline, $4 \%$ of lysophosphatidyl choline) were gift samples from Lipoid GmbH (Newark, USA), sugar spheres and sugar was obtained from RA Chem Pharma Limited (Hyderabad, India). Lipsin Retard marketed by Solvay Pharmaceuticals, Inc. All other reagents used were of analytical grade.

\section{Methods}

Drug loading: Drug was loaded onto sugar spheres using fluidized bed processor (Model Glatt FBE-250). The sugar spheres were loaded into the fluidized bed processor and warmed for about $10-15$ minutes. Binder solution was prepared by dissolving Povidone K30 and sugar in purified water under constant stirring. Unmicronized fenofibrate which was pre blended and pulverized $(0.5 \mathrm{~mm}$ screen pulverizer) with other excipients was incorporated intermittently into the solution under constant stirring. The obtained solution was loaded onto the circulating sugar spheres $(800 \mu \mathrm{m})$. The wet spheres were dried simultaneously during the process of circulation in wurster chamber. The process was continued till the complete solution was consumed. The various processing variables namely inlet air temperature (40$45^{\circ} \mathrm{C}$ ), outlet air temperature $\left(30-33^{\circ} \mathrm{C}\right)$, product temperature $(36$ $\left.40^{\circ} \mathrm{C}\right)$, chamber humidity $(58-60 \%)$, air flow $\left(85 \mathrm{~m}^{3} / \mathrm{hr}\right)$, compressed air pressure (1.5-3.0 kg/cm²) The peristaltic pump rpm (10-35) and spray rate $(2 \mathrm{ml} / \mathrm{min})$ were optimized in the preliminary trials.

Design of experiment to optimize drug loaded fenofibrate pellets: Taguchi L16 design of experiment was used to study the effect of two variables namely, type of solubilizer and concentration of solubilizer at four different levels as given in Table 1 and Table 2. All the experimental runs were analyzed using Minitab statistical software package (version 15).

\section{Characterization methods}

Infrared Spectroscopy analysis of drug and excipients: FT-IR spectra were obtained using FT-IR spectrometer (Model Nicolet 5700) by the conventional $\mathrm{KBr}$ pellet method. The samples were grounded gently with anhydrous $\mathrm{KBr}$ and compressed to form pellet. Scanning range was $400-4000 \mathrm{~cm}^{-1}$ and the resolution was $4 \mathrm{~cm}^{-1}$ (Table 3).

Sieve analysis and usable yield or \% theoretical yield of pellets: Auchi experimental runs were evaluated for particle size distribution using standard set of sieves and were shaken using RoTap sieve shaker (Model RX-29) for about 20 minutes. The formulations for which not less than $90 \%$ of pellets passed through \#16 (1180 $\mu \mathrm{m})$ and not less

\begin{tabular}{|l|l|l|l|l|}
\hline Dependent Variables & Level 1 & Level 2 & Level 3 & Level 4 \\
\hline $\begin{array}{l}\text { Factor A: Type of } \\
\text { solubilizer }\end{array}$ & AT & SPC 70 & PL 80H & PL 90H \\
\hline $\begin{array}{l}\text { Factor B: Concentration } \\
\text { of solubilizer }\end{array}$ & $0.5 \%$ & $1 \%$ & $1.5 \%$ & $2 \%$ \\
\hline
\end{tabular}

Table 1: Taguchi L16 $\left(2^{4}\right)$ orthogonal array design of experiment. than $90 \%$ of pellets retained on \#20 $(850 \mu \mathrm{m})$ were selected for further studies and were weighed after drug loading. The theoretical yield was calculated respectively.

Mechanical Crushing force: At least 20 pellets from the usable yield fraction of each formulation were evaluated for their diametrical crushing force using a tablet strength tester (Electrolab EH 01) [8].

Abrasion Resistance : A pre-weighed sample (approximately 10 g) taken from the usable yield fraction was placed in an abrasion drum (Vankel Industries Inc. USA) that was configured to raise and drop the pellets from $200 \mathrm{~mm}$. The stress levels on pellets were enhanced by adding $1 \mathrm{~mm}$ glass beads. After 100 revolutions at $25 \mathrm{rpm}$, the mass retained on the sieve $(1190 \mu \mathrm{m})$ was weighed and the abrasion resistance was calculated as the percentage loss of mass between initial and final weights of each pellet batch. Each batch was analyzed in triplicate.

Residual moisture: The residual water content present in the pellets after drying was determined by United States Pharmacopeia (USP) method using Karl Fischer titrator. The equipment was precalibrated and standardized with disodium tartarate dihydrate. Pellets, approximately $250 \mathrm{mg}$ were accurately weighed and immediately placed in the moisture analyzer for titration with Karl Fischer reagent. Each batch was analyzed in triplicate.

Flow Properties: Bulk and tapped density of pellets was determined using USP density apparatus. The bulk density, tapped densities were determined initially from which Hausner's ratio was calculated. The angle of repose was determined using fixed funnel method [9].

\section{Assay of the prepared pellets}

High performance liquid Chromatography (HPLC) method of analysis: The HPLC system consisted LC-10AT pump system, an auto injector SIL-10A (Shimadzu) at a flow rate of $1.0 \mathrm{ml} / \mathrm{min}$ of mobile phase, peak detection by UV detector (SPD-10AV) at wavelength 286 $\mathrm{nm}$. A reverse phase column Hypersil BDS C18, $150 \mathrm{~mm}$ X $4.6 \mathrm{~mm}$ kept at $25^{\circ} \mathrm{C}$ by a column oven with $5 \mu \mathrm{m}$ particle size was used as stationary phase. A mixture of $30 \%$ of orthophosphoric acid buffer in $70 \%$ of acetonitrile $(30: 70 \mathrm{v} / \mathrm{v})$ was used as mobile phase. The injection volume was $20 \mu \mathrm{l}$. The samples were detected at $286 \mathrm{~nm}$ and integrated using the RF 10A (version 1.1) LC Solutions software program. Samples were diluted with mobile phase before injection and run for 12 minutes.

In vitro dissolution study of prepared pellets: Accurately weighed amount of pellets equivalent quantity of $250 \mathrm{mg}$ fenofibrate were filled into size ' 1 ' capsules and the dissolution was performed in $1000 \mathrm{ml}$ of $0.1 \mathrm{M}$ Sodium lauryl sulfate in water as biorelevant dissolution medium, using USP Type II (paddle) apparatus (Electrolab) at temperature of 37 $\pm 0.5^{\circ} \mathrm{C}$ for 8 hours. Aliquots of $5 \mathrm{ml}$ were withdrawn for every one hour and were replaced with fresh medium. The samples were analyzed spectrophotometrically (UV 2450) at maximum wavelength $\left(\lambda_{\max }\right)$ of drug at $291.0 \mathrm{~nm}$ against the medium as the blank. For each dissolution run, a mean of six determinations was recorded.

\section{Pharmacokinetic study in rats}

The pharmacokinetics of drug and test (AT 1\% and PL 90H 2\%) was evaluated following oral administration. The study was conducted at G. Pulla Reddy College of Pharmacy, Hyderabad, India as per the regulations specified by the Institutional Animal Ethics Committee and Committee for the Purpose of Control and Supervision of Experiments 
Citation: Indira TK, Lakshmi PK, Balasubramaniam J, Rajesh YV (2012) Enhancement of Bioavailability of Fenofibrate with Alpha Tocopherol and Phospholipids as Solubilizers. J Bioequiv Availab S14. doi:10.4172/jbb.S14-006

Page 3 of 8

\begin{tabular}{|c|c|c|c|c|c|c|c|c|}
\hline Material $(\% w / w)$ & Run1 & Run2 & Run 3 & Run 4 & Run 5 & Run 6 & Run7 & Run8 \\
\hline Fenofibrate (unmicronized) & $75 \%$ & $75 \%$ & $75 \%$ & $75 \%$ & $75 \%$ & $75 \%$ & $75 \%$ & $75 \%$ \\
\hline HPMC-E5 & $0.06 \%$ & $0.06 \%$ & $0.06 \%$ & $0.06 \%$ & $0.06 \%$ & $0.06 \%$ & $0.06 \%$ & $0.06 \%$ \\
\hline HPMC-K4M & $0.03 \%$ & $0.03 \%$ & $0.03 \%$ & $0.03 \%$ & $0.03 \%$ & $0.03 \%$ & $0.03 \%$ & $0.03 \%$ \\
\hline PVP-K30 & $1.33 \%$ & $1.33 \%$ & $1.33 \%$ & $1.33 \%$ & $1.33 \%$ & $1.33 \%$ & $1.33 \%$ & $1.33 \%$ \\
\hline Sugar Spheres & $20.28 \%$ & $19.78 \%$ & & & $20.28 \%$ & $19.78 \%$ & $19.28 \%$ & $18.78 \%$ \\
\hline Vitamin-E & $0.5 \%$ & $1 \%$ & $1.5 \%$ & $2 \%$ & - & - & - & - \\
\hline Soya Phosphatidyl Choline 70 & - & - & - & - & $0.5 \%$ & $1 \%$ & $1.5 \%$ & $2 \%$ \\
\hline Phospholipon - $80 \mathrm{H}$ & - & - & - & - & - & - & - & - \\
\hline Phospholipon - 90H & - & - & - & - & - & - & - & - \\
\hline \multicolumn{9}{|l|}{ Binder Solution } \\
\hline PVP-K30 & $1.0 \%$ & $1.0 \%$ & $1.0 \%$ & $1.0 \%$ & $1.0 \%$ & $1.0 \%$ & $1.0 \%$ & $1.0 \%$ \\
\hline Sugar & $1.8 \%$ & $1.8 \%$ & $1.8 \%$ & $1.8 \%$ & $1.8 \%$ & $1.8 \%$ & $1.8 \%$ & $1.8 \%$ \\
\hline Water & q.s. & q.s. & q.s. & q.s. & q.s. & q.s. & q.s. & q.s. \\
\hline
\end{tabular}

Table 2: Taguchi experimental runs formulae.

\begin{tabular}{|c|c|c|c|c|c|c|c|c|}
\hline Material (\%w/w) & Run9 & Run10 & Run11 & Run12 & Run13 & Run14 & Run15 & Run16 \\
\hline Fenofibrate (unmicronized) & $75 \%$ & $75 \%$ & $75 \%$ & $75 \%$ & $75 \%$ & $75 \%$ & $75 \%$ & $75 \%$ \\
\hline HPMC-E5 & $0.06 \%$ & $0.06 \%$ & $0.06 \%$ & $0.06 \%$ & $0.06 \%$ & $0.06 \%$ & $0.06 \%$ & $0.06 \%$ \\
\hline HPMC-K4M & $0.03 \%$ & $0.03 \%$ & $0.03 \%$ & $0.03 \%$ & $0.03 \%$ & $0.03 \%$ & $0.03 \%$ & $0.03 \%$ \\
\hline PVP-K30 & $1.33 \%$ & $1.33 \%$ & $1.33 \%$ & $1.33 \%$ & $1.33 \%$ & $1.33 \%$ & $1.33 \%$ & $1.33 \%$ \\
\hline Sugar spheres & $20.28 \%$ & $19.78 \%$ & $19.28 \%$ & $18.78 \%$ & $20.28 \%$ & $19.78 \%$ & $19.28 \%$ & $18.78 \%$ \\
\hline Vitamin-E & - & - & - & - & - & - & - & - \\
\hline Soya phosphatidyl choline 70 & - & - & - & - & - & - & - & - \\
\hline Phospholipon $80 \mathrm{H}$ & $0.5 \%$ & $1.0 \%$ & $1.5 \%$ & $2 \%$ & - & - & - & - \\
\hline Phospholipon $90 \mathrm{H}$ & - & - & - & - & $0.5 \%$ & $1.0 \%$ & $1.5 \%$ & $2.0 \%$ \\
\hline \multicolumn{9}{|l|}{ Binder Solution } \\
\hline PVP-K30 & $1.0 \%$ & $1.0 \%$ & $1.0 \%$ & $1.0 \%$ & $1.0 \%$ & $1.0 \%$ & $1.0 \%$ & $1.0 \%$ \\
\hline Sugar & $1.8 \%$ & $1.8 \%$ & $1.8 \%$ & $1.8 \%$ & $1.8 \%$ & $1.8 \%$ & $1.8 \%$ & $1.8 \%$ \\
\hline Water & q.s. & q.s. & q.s. & q.s. & q.s. & q.s. & q.s. & q.s. \\
\hline
\end{tabular}

Table 3: Taguchi experimental runs formulae.

\begin{tabular}{|c|c|c|c|c|c|c|c|c|c|}
\hline Experimental Run & $\%$ TYP & Crushing force & Abrasion & Moisture & Assay & Bulk Density & Tapped Density & Hausner's ratio & Angle of repose \\
\hline Run $1_{1}^{a}$ & $95.1 \pm 0.01$ & $6.1 \pm 0.07$ & $0.55 \pm 0.07$ & $2.05 \pm 0.07$ & $96.02 \pm 0.01$ & $0.802 \pm 0.01$ & $0.806 \pm 0.01$ & $1.05 \pm 0.07$ & $25.25 \pm 0.35$ \\
\hline Run $2^{\mathrm{a}}$ & $98.03 \pm 0.08$ & $6.45 \pm 0.49$ & $0.65 \pm 0.07$ & $2.2 \pm 0.28$ & $98.43 \pm 0.007$ & $0.804 \pm 0.002$ & $0.809 \pm 0.02$ & $1.00 \pm 0.007$ & $25.2 \pm 0.14$ \\
\hline Run $3_{3}^{\mathrm{a}}$ & $97.7 . \pm 0.16$ & $6.2 \pm 0.14$ & $0.7 \pm 0.14$ & $2.25 \pm 0.21$ & $97.4 \pm 0.21$ & $0.781 \pm 0.001$ & $0.787 \pm 0.002$ & $1.00 \pm 0.004$ & $25.3 \pm 0.42$ \\
\hline Run 4a ${ }_{4}^{a}$ & 90.540 .18 & $6.25 \pm 0.21$ & $0.8 \pm 0.14$ & $2.4 \pm 0.07$ & $99.08 \pm 0.28$ & $0.797 \pm 0.001$ & $0.803 \pm 0.002$ & $1.00 \pm 0.004$ & $25.5 \pm 0.28$ \\
\hline Run $5^{\mathrm{b}}{ }_{1}$ & $95.2 \pm 0.06$ & $6.35 \pm 0.07$ & $0.575 \pm 0.03$ & $2.15 \pm 0.07$ & $95.9 \pm 0.03$ & $0.792 \pm 0.002$ & $0.797 \pm 0.002$ & $1.00 \pm 0.008$ & $25.6 \pm 0.28$ \\
\hline Run $6^{b}{ }_{2}$ & $96.4 \pm 0.015$ & $6.3 \pm 0.28$ & $0.55 \pm 0.0$ & $2.3 \pm 0.14$ & $97.54 \pm 0.15$ & $0.793 \pm 0.007$ & $0.796 \pm 0.002$ & $1.00 \pm 0.004$ & $25.55 \pm 0.35$ \\
\hline Run $7_{3}^{\mathrm{b}}$ & $97.2 \pm 0.42$ & $6.35 \pm 0.21$ & $0.675 \pm 0.03$ & $2.32 \pm 0.11$ & $98.13 \pm 0.001$ & $0.794 \pm 0.001$ & $0.798 \pm 0.007$ & $1.00 \pm 0.002$ & $25.25 \pm 0.21$ \\
\hline Run $8^{\mathrm{b}}{ }_{4}$ & $96.7 \pm 0.02$ & $6.1 \pm 0.07$ & $0.82 \pm 0.014$ & $2.29 \pm 0.07$ & $95.76 \pm 0.06$ & $0.793 \pm 0.007$ & $0.797 \pm 0.002$ & $1.00 \pm 0.002$ & $25.45 \pm 0.21$ \\
\hline Run $9^{c}{ }_{1}$ & $97.2 \pm 0.28$ & $6.25 \pm 0.21$ & $0.58 \pm 0.06$ & $2.47 \pm 0.03$ & $98.07 \pm 0.02$ & $0.792 \pm 0.007$ & $0.796 \pm 0.001$ & $1.00 \pm 0.008$ & $25.5 \pm 0.28$ \\
\hline Run $10_{2}^{c}$ & $96.8 \pm 0.09$ & $6.4 \pm 0.14$ & $0.7 \pm 0.14$ & $2.42 \pm 0.1$ & $97.71 \pm 0.008$ & $0.794 \pm 0.002$ & $0.796 \pm 0.002$ & $1.00 \pm 0.002$ & $25.3 \pm 0.00$ \\
\hline Run $11^{\mathrm{c}}{ }_{3}$ & $95.6 \pm 0.042$ & $6.22 \pm 0.17$ & $0.57 \pm 0.028$ & $2.45 \pm 0.07$ & $96.41 \pm 0.04$ & $0.795 \pm 0.001$ & $0.798 \pm 0.001$ & $1.00 \pm 0.001$ & $25.55 \pm 0.07$ \\
\hline Run $12^{\mathrm{c}}{ }_{4}$ & $95.3 \pm 0.01$ & $6.22 \pm 0.17$ & $0.635 \pm 0.02$ & $2.3 \pm 0.14$ & $97.35 \pm 0.41$ & $0.792 \pm 0.002$ & $0.797 \pm 0.001$ & $1.00 \pm 0.004$ & $25.45 \pm 0.35$ \\
\hline Run $13^{\mathrm{d}}{ }_{1}$ & $97.7 \pm .0 .028$ & $6.37 \pm 0.03$ & $0.72 \pm 0.02$ & $2.39 \pm 0.07$ & $98.1 \pm 0.21$ & $0.791 \pm 0.00$ & $0.795 \pm 0.007$ & $1.00 \pm 0.008$ & $25.2 \pm 0.14$ \\
\hline Run $14_{2}^{\mathrm{d}}$ & $96.3 \pm 0.007$ & $6.42 \pm 0.10$ & $0.63 \pm 0.07$ & $2.32 \pm 0.18$ & $97.47 \pm 0.14$ & $0.792 \pm 0.002$ & $0.797 \pm 0.007$ & $1.00 \pm 0.001$ & $25.6 \pm 0.42$ \\
\hline Run $15^{\mathrm{d}}{ }_{3}$ & $97.6 \pm 0.14$ & $6.25 \pm 0.21$ & $0.57 \pm 0.07$ & $2.34 \pm 0.21$ & $97.54 \pm 0.07$ & $0.795 \pm 0.002$ & $0.799 \pm 0.007$ & $1.00 \pm 0.001$ & $25.05 \pm 0.07$ \\
\hline Run $16^{\mathrm{d}}{ }_{4}$ & $98.5 \pm 0.05$ & $6.4 \pm 0.14$ & $0.71 \pm 0.04$ & $2.42 \pm 0.03$ & $98.75 \pm 0.01$ & $0.795 \pm 0.001$ & $0.805 \pm 0.007$ & $1.00 \pm 0.01$ & $25.5 \pm 0.28$ \\
\hline
\end{tabular}

Table 4: Comparative characterized properties of Taguchi experimental runs $(n=3)$ (Mean \pm S.D).

on Animals (CPSEA). In total 18 (6 per group) male Wistar rats (6-7 weeks old) weighing between 180-230 g were used for the study. All rats had free access to tap water and pelleted diet (Ssniff rats pellet food, Ssniff Spezialdiaten, Germany). The rats were housed in a cage and maintained on a $12 \mathrm{~h}$ light/dark at room temperature $\left(21^{\circ} \mathrm{C}\right.$ to $24^{\circ} \mathrm{C}$ ) and relative humidity of 50 to $70 \%$ and acclimatized to study area conditions for atleast 5 days before dosing. General and environmental conditions were strictly monitored. The room underwent 10 fresh air change cycles per hour. Rats were implanted with canula in the jugular vein for blood sampling. The surgery was performed two days before dosing under anesthesia. The animals were fasted at least $10 \mathrm{~h}$ prior to dose administration and for 4 hours post dose with free access to water. Individual oral doses of the test and reference samples were prepared $(10 \mathrm{mg} / \mathrm{kg}$ free base) and accurately weighed drug material was carefully transferred into the dosing syringe containing aliquot of gelatin gel. Transfer the sample into the syringe barrel was accomplished either using a butter-paper funnel/with a spatula; the funnel was weighed before and after transferring drug to account for any loss by sticking to 
Citation: Indira TK, Lakshmi PK, Balasubramaniam J , Rajesh YV (2012) Enhancement of Bioavailability of Fenofibrate with Alpha Tocopherol and Phospholipids as Solubilizers. J Bioequiv Availab S14. doi:10.4172/jbb.S14-006

Page 4 of 8

funnel. Separate funnels were used to prepare each dose. After transfer of the drug material into the syringe, an aliquot of gelatin was placed on top of the drug powder, thus effectively sandwiching it between 2 layers of gelatin. The sample was attached to an oral feeding needle and administered into the stomach. After dosing, syringe was rinsed with $1 \mathrm{~mL}$ of water and dosed again. Serial blood samples $(50 \mu \mathrm{L})$ were withdrawn from the cannulated jugular vein at: Pre dose, 1, 2, 4, 6, 8, 9 and $24 \mathrm{~h}$ post-dosing and collected in labeled tubes containing $20 \mu \mathrm{L}$ of EDTA dipotassium dehydrate solution $(200 \mathrm{mM})$ per $\mathrm{ml}$ of blood as anticoagulant. Blood samples were held on ice until centrifuged at $10000 \mathrm{rpm} ; 4^{\circ} \mathrm{C}$ for $10 \mathrm{~min}$. Plasma was transferred to individual Eppendorf tubes and stored below $-70^{\circ} \mathrm{C}$ until bioanalysis.

\section{Bioanalysis}

The samples were analyzed by High performance liquid chromatography (HPLC Model no: API LC-1018, Shimadzu, Japan) by using reverse phase column (Hypersil BDS C18 $150 \mathrm{~mm} \times 4.6 \mathrm{~mm}$ ) and UV detector at $286 \mathrm{~nm}$ using 30:70 orthophosphoric acid buffer and acetonitrile. Clofibric acid stock solution was used as the internal standard. Under these conditions, no interference was observed for both samples and pure drug. The standard curve was linear from 1ng/ $\mathrm{ml}$ to $2000 \mathrm{mg} / \mathrm{ml}$.

\section{Pharmacokinetic data analysis}

The area under the drug concentration-time curve from zero to $24 \mathrm{~h}\left(\mathrm{AUC}_{0 \rightarrow 24 \mathrm{~h}}\right.$ ) and Mean Residence Time (MRT) were calculated using noncompartmental analysis (WinNonlin 2.1, Pharsight Corp., Mountain View, CA). The maximal plasma concentration of drug $\left(\mathrm{C}_{\max }\right)$ and the time to reach maximum plasma concentration $\left(\mathrm{T}_{\max }\right)$ were directly obtained from plasma data and area under the curve (AUC), elimination rate constant $\left(\mathrm{K}_{\mathrm{E}}\right)$ and half life $\left(\mathrm{t}_{1 / 2}\right)$, were also computed.

\section{Results and Discussion}

\section{Infrared Spectroscopy analysis of drug and excipient mixture}

The principal peaks of pure fenofibrate were observed at 1729.4, 1651.0, 1598.9 and $925.1 \mathrm{~cm}^{-1}$.The characteristic peaks for drug and excipients mixture were found at 1729.6, 1650.1, and 1599.1 and $925.5 \mathrm{~cm}^{-1}$. No peaks were found at these wave numbers for excipients indicating no possible interaction between drug and the polymer therein (Figure 1).

\section{Characterization of taguchi experimental runs}

The characterized properties for Taguchi runs given in Table 4 suggests that the two factors under consideration namely type and concentration of solubilizers have insignificant effect on the properties of $\%$ theoretical yield, mechanical crushing force, abrasion resistance, residual moisture, assay and flow properties.

\section{In vitro release studies}

The in vitro dissolution profile of Taguchi experimental runs indicates that for Run 1 to Run 2 with $0.5 \%, 1 \%$ alpha tocopherol and Run 5 to Run 16 with $0.5 \%, 1 \%, 1.5 \%, 2 \%$ of soy phosphatidyl choline 70, Phospholipon $80 \mathrm{H}$ and Phospholipon $90 \mathrm{H}$ the release was acceptable with $10-30 \%$ of release in first hour, $50-75 \%$ drug in fourth hour and greater than $75 \%$ of release at the end of seventh hour. While for Run 3 and Run 4 containing 1.5\% and 2\% alpha tocopherol, the release was greater than $30 \%$ in first hour and greater than $90 \%$ of drug was released at the end of seventh hour (Table 5).

The statistical analysis (Figure 2a) suggests that factor A, type of solubilizer has pronounced and significant effect on dissolution behavior, since the obtained $\mathrm{P}$ value is $0.020(\mathrm{P}<0.05)$. The effect can be indicated in descending order as

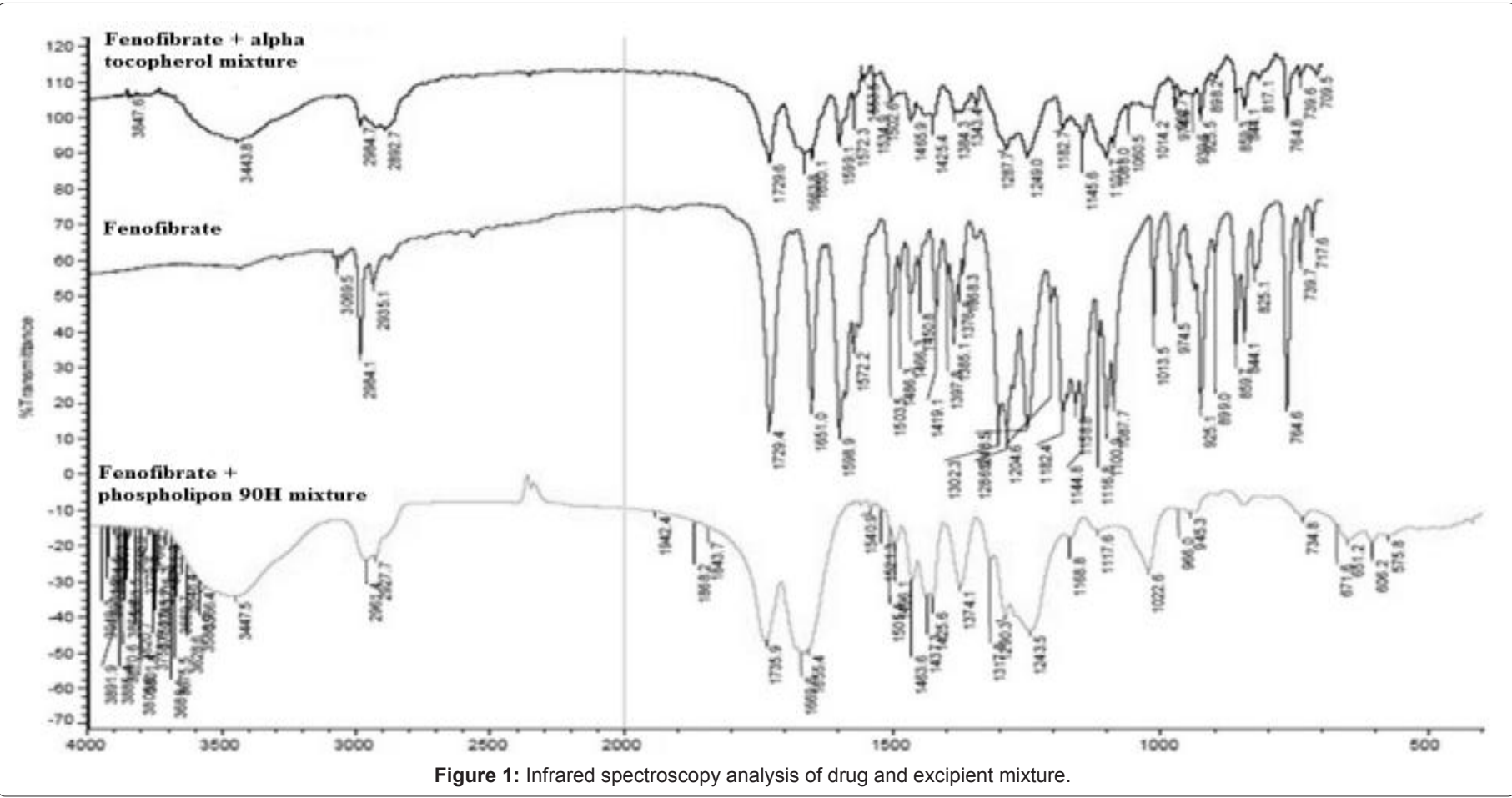


Citation: Indira TK, Lakshmi PK, Balasubramaniam J , Rajesh YV (2012) Enhancement of Bioavailability of Fenofibrate with Alpha Tocopherol and Phospholipids as Solubilizers. J Bioequiv Availab S14. doi:10.4172/jbb.S14-006

Page 5 of 8

\begin{tabular}{|c|c|c|c|c|c|c|c|c|}
\hline \multirow{2}{*}{ Experimental Run } & \multicolumn{8}{|c|}{ Time in hours } \\
\hline & $1 \mathrm{hr}$ & $2 \mathrm{hr}$ & $3 \mathrm{hr}$ & $4 \mathrm{hr}$ & $5 \mathrm{hr}$ & $6 \mathrm{hr}$ & $7 \mathrm{hr}$ & $8 \mathrm{hr}$ \\
\hline Run 1 & $23.5 \pm 0.01$ & $39.14 \pm 0.21$ & $52.02 \pm 0.03$ & $60.9 \pm 0.14$ & $69 \pm 0.13$ & $74.1 \pm 0.028$ & $80 \pm 0.71$ & $84.4 \pm 0.04$ \\
\hline Run 2 & $27 \pm 0.007$ & $44.31 \pm 0.15$ & $57.87 \pm 0.17$ & $69.65 \pm 0.07$ & $78.6 \pm 0.06$ & $86.7 \pm 0.03$ & $92.4 \pm 0.51$ & $97.7 \pm 0.007$ \\
\hline Run 3 & $43.9 \pm 0.14$ & $63.57 \pm 0.04$ & $76.29 \pm 0.009$ & $85.6 \pm 0.28$ & $90.8 \pm 0.08$ & - & - & - \\
\hline Run 4 & $45.7 \pm 0.21$ & $65.36 \pm 0.04$ & $78.08 \pm 0.02$ & $87.7 \pm 0.14$ & $94.6 \pm 0.007$ & - & - & - \\
\hline Run 5 & $25.5 \pm 0.28$ & $34.95 \pm 0.21$ & $45.79 \pm 0.01$ & $55.03 \pm 0.04$ & $61.2 \pm 0.28$ & $68 \pm 0.42$ & $72.2 \pm .0 .009$ & $76 \pm .0 .028$ \\
\hline Run 6 & $28.5 \pm 0.56$ & $37.56 \pm 0.04$ & $53.26 \pm 0.02$ & $66.9 \pm 0.14$ & $76.7 \pm 0.14$ & $83.62 \pm 0.02$ & $97.8 \pm 0.14$ & $99.3 \pm 0.015$ \\
\hline Run 7 & $25.07 \pm 0.03$ & $39.92 \pm 0.03$ & $51.75 \pm 0.35$ & $61.5 \pm 0.7$ & $67.5 \pm 0.42$ & $75.14 \pm 0.05$ & $81.1 \pm 0.31$ & $86.2 \pm 0.01$ \\
\hline Run 8 & $23.42 \pm 0.03$ & $39.8 \pm 0.02$ & $51.25 \pm 0.06$ & $60.68 \pm 0.02$ & $67.4 \pm 0.28$ & $75.2 \pm 0.04$ & $80.2 \pm 0.21$ & $85.8 \pm 0.06$ \\
\hline Run 9 & $17.2 \pm 0.04$ & $25.29 \pm 0.01$ & $39.7 \pm 0.14$ & $50.2 \pm 0.17$ & $60.14 \pm 0.05$ & $68.3 \pm 0.01$ & $80.8 \pm 0.42$ & $88.7 \pm 0.01$ \\
\hline Run 10 & $18.1 \pm 0.02$ & $27.1 \pm 0.007$ & $42.8 \pm 0.14$ & $52.6 \pm 0.08$ & $62.5 \pm 0.32$ & $70.44 \pm 0.44$ & $82.16 \pm 0.5$ & $91.7 \pm 0.16$ \\
\hline Run 11 & $19.1 \pm 0.41$ & $29.4 \pm 0.21$ & $45.07 \pm 0.42$ & $56.14 \pm 0.01$ & $64.7 \pm 0.06$ & $73.21 \pm 0.007$ & $84.14 \pm 0.28$ & $93.4 \pm 0.32$ \\
\hline Run 12 & $23.7 \pm 0.21$ & $39.9 \pm 0.09$ & $53.6 \pm 0.31$ & $64.3 \pm 0.007$ & $73.2 \pm 0.12$ & $79.4 \pm 0.56$ & $85.3 \pm 0.06$ & $89.5 \pm 0.18$ \\
\hline Run 13 & $22.08 \pm 0.14$ & $31.3 \pm 0.042$ & $42.08 \pm 0.02$ & $54.7 \pm 0.01$ & $62.1 \pm 0.10$ & $71.9 \pm 0.10$ & $84.2 \pm 0.02$ & $92 . \pm 0.16$ \\
\hline Run 14 & $24.7 \pm 0.07$ & $33.78 \pm 0.28$ & $44.5 \pm 0.01$ & $58.8 \pm 0.42$ & $64.3 \pm 0.21$ & $73.2 \pm 0.14$ & $89.4 \pm 0.21$ & $94.3 \pm 0.06$ \\
\hline Run 15 & $26.8 \pm 0.01$ & $35.8 \pm 0.15$ & $46.8 \pm 0.27$ & $62.04 \pm 0.15$ & $66.7 \pm 0.14$ & $75.6 \pm 0.01$ & $93.6 \pm 0.28$ & $96.8 \pm 0.15$ \\
\hline Run 16 & $21.2 \pm 0.08$ & $36.21 \pm 0.1$ & $52.13 \pm 0.12$ & $64.53 \pm 0.19$ & $75.32 \pm 0.28$ & $84.7 \pm 0.07$ & $94.47 \pm 0.12$ & $98.42 \pm 0.08$ \\
\hline
\end{tabular}

Table 5: In vitro dissolution data for Taguchi experimental runs $(n=6)$

A) Box plot for effect of solubilizers on in vitro dissolution of drug

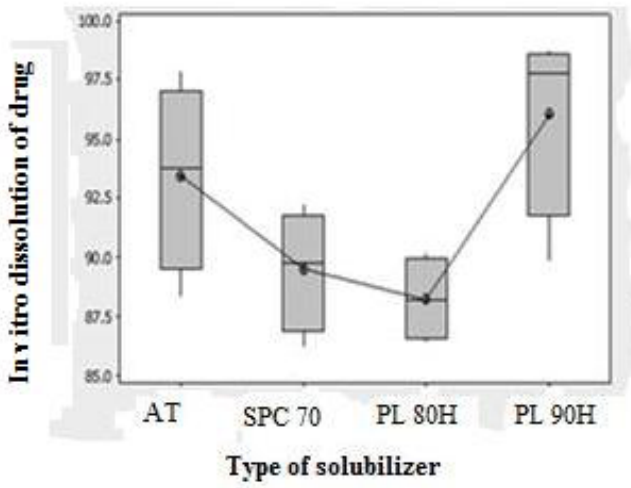

B)

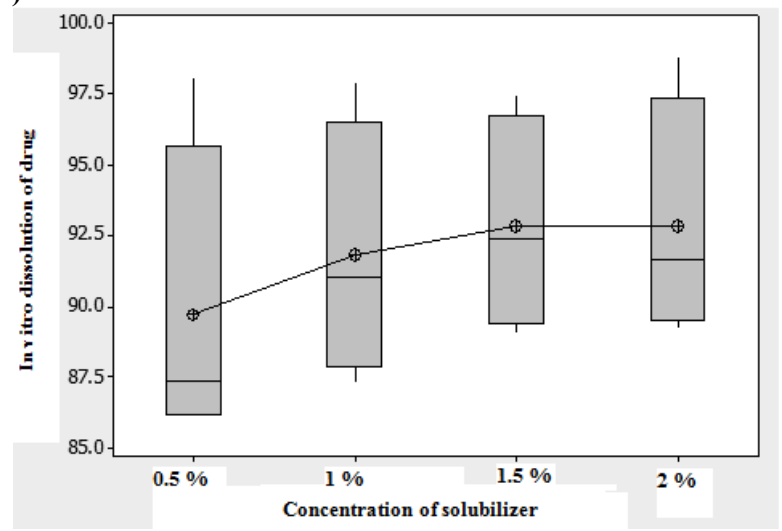

Figure 2: Box plot for effect of (a) type of solubilizer and (b) concentration of solubilizer on the in vitro dissolution behavior of drug.
Phospholipon $90 \mathrm{H}>$ alpha tocopherol $>$ soy phosphatidyl choline $70>$ Phospholipon $80 \mathrm{H}$

While concentration of solubilizer (Figure 2b) suggests no pronounced effect on dissolution behavior of drug in lipid phase, since obtained $\mathrm{P}$ value is $0.756,(\mathrm{P}>0.05)$ increase in concentration suggested enhanced dissolution behavior of drug.

Comparative dissolution profiles for Taguchi optimized runs with that of marketed are elucidated (Figure 3).

\section{Pharmacokinetic parameters}

The comparative average serum concentration-time profiles of fenofibrate following oral administration of $10 \mathrm{mg} / \mathrm{kg}$ in the rat (Figure $4)$.

\section{Statistical Analysis of data}

ANOVA results were obtained using the Minitab statistical software package (version 15). The response factor or dependent factor studied was dissolution behavior of drug. The effect of two parameters effect of solubilizer and concentration of solubilizer on the in vitro dissolution of drug were studied. The F, P values obtained was $4.81,0.020$ when the response was compared with factor $\mathrm{A}$, namely type of solubilizer, the obtained F, P values when the response was compared with factor B, namely concentration of solubilizer were 0.40 and 0.756 respectively. The tabulated $\mathrm{F}$ value with three degrees of freedom in the numerator and denominator is 15.4. Therefore the variances cane be considered significantly indifferent and the $\mathrm{P}<0.05$ when the response was studied with factor B stating that factor B has significant effect on the response variable. While $\mathrm{P}>0.05$ when the response was studied against factor A stating that factor $\mathrm{A}$ has insignificant effect on the response variable.

\section{Model independent methods}

Model independent methods were used to compute the Mean Dissolution Time (MDT) and also to compare Taguchi experimental 
Citation: Indira TK, Lakshmi PK, Balasubramaniam J , Rajesh YV (2012) Enhancement of Bioavailability of Fenofibrate with Alpha Tocopherol and Phospholipids as Solubilizers. J Bioequiv Availab S14. doi:10.4172/jbb.S14-006

Page 6 of 8

runs to the marketed formulation by calculating similarity $\left(f_{2}\right)$ and difference factor $\left(f_{1}\right)$ that signifies the Taguchi experimental runs for acceptance or rejectance for further studies. The computed values are given in Table 6.

\section{Test for Comparison of means of reference and optimized Taguchi experimental runs}

Two formulations of Taguchi experimental runs were optimized based on characterized properties, ANOVA, release kinetics, mean dissolution time, similarity and difference factor. Of all the Taguchi experimental runs, Run $2^{\mathrm{a}}{ }_{2}$ with $1 \%$ alpha -tocopherol and Run $16^{\mathrm{d}}$ with $2 \%$ Phospholipon $90 \mathrm{H}$ were proved to be the optimized runs with respective to highest similarity factor and highest drug release. Therefore the two formulations were compared with reference for statistical significance. For this, a two-sided Paired-Sample ' $t$ ' was applied. The obtained values were compared with standard tables for significance at confidence intervals of $95 \%$ [10]. The summary of test is briefed out in Table 7 .

The resultant ' $t$ ' was found to be 5.200626976 for Run $2^{\mathrm{a}}{ }_{2}$ and was found to be 4.28116546 for Run $16^{\mathrm{d}}{ }_{4}$ For a two sided test at $5 \%$ level, a $t$ value of 2.36 is needed for significance with seven degrees of freedom (Since $\mathrm{n}=8$ ). Therefore the difference is significant at $5 \%$ level, stating that the optimized formulations have better bioavailability compared to marketed, which has already been evidenced from (Figure 3), (Table 7) states that Run $2^{\mathrm{a}}{ }_{2}$ to be more bioavailable at low concentration.

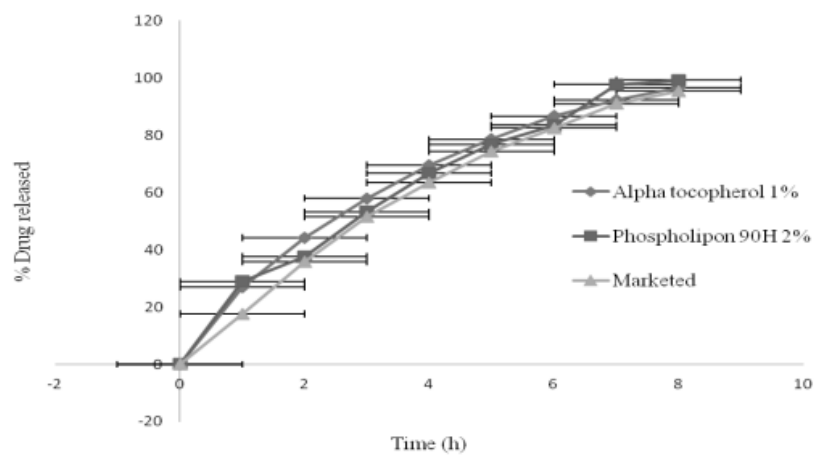

Figure 3: Comparative release profiles of optimized Taguchi runs with marketed formulation.

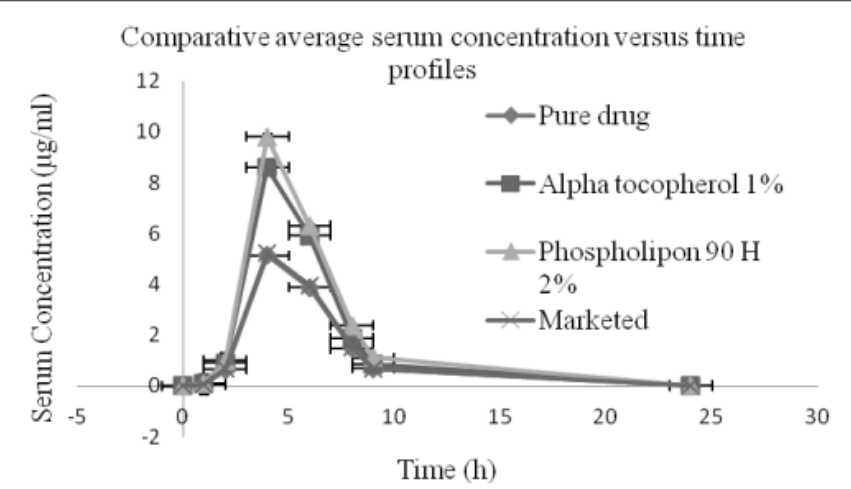

Figure 4: Comparative average serum concentration-time profiles of Taguchi optimized runs with marketed formulation and pure drug.

\begin{tabular}{|c|c|c|c|}
\hline \multirow[b]{2}{*}{ Experimental Runs } & \multicolumn{3}{|c|}{ Calculation of MDT, $f_{1}$ and $f_{2}$ for the dissolution data } \\
\hline & MDT & $f_{2}$ & $\mathrm{f}_{1}$ \\
\hline Run $1_{1}^{\mathrm{a}}$ & 0.001953 & 57.31 & 9.47 \\
\hline $\operatorname{Run} 2_{2}^{\mathrm{a}}{ }_{2}$ & 0.00172479 & 61.19 & 8.11 \\
\hline Run $3_{3}^{\text {a }}$ & 0.757755 & 31.2 & 48.2 \\
\hline Run $4_{4}^{\mathrm{a}}$ & 0.674866 & 29.3 & 53 \\
\hline Run $5^{b}$ & 0.00100709 & 45.19 & 17.17 \\
\hline Run $6^{\mathrm{b}}{ }_{2}$ & 0.00218616 & 60.01 & 6.26 \\
\hline Run $7^{\mathrm{b}}{ }_{3}$ & 0.001483015 & 58.09 & 9.39 \\
\hline Run $8_{4}^{\mathrm{b}}$ & 0.00202604 & 57.98 & 9.39 \\
\hline Run $9^{c}{ }_{1}$ & 0.014877 & 47.56 & 68.01 \\
\hline Run $10^{c}{ }_{2}$ & 0.0123761 & 52.01 & 12.83 \\
\hline Run $11^{c}$ & 0.0100919 & 58.03 & 9.73 \\
\hline Run $12^{\mathrm{c}}{ }_{4}$ & 0.002579166 & 59.8 & 9.5 \\
\hline Run $13^{d}{ }_{1}$ & 0.005655444 & 54.03 & 11.75 \\
\hline Run $14^{\mathrm{d}}{ }_{2}$ & 0.003653 & 59.63 & 8.44 \\
\hline Run $15^{d}{ }_{3}$ & 0.00267744 & 63.32 & 6.65 \\
\hline Run $16^{d}{ }_{4}^{*}$ & 0.0072691 & 80.48 & 2.925 \\
\hline Marketed & 0.011559507 & - & - \\
\hline
\end{tabular}

Table 6: Descriptive statistical model independent methods for calculation of MDT, $f_{1}$ and $f_{2}$ for the dissolution data $(n=6)$.

Model independent methods suggests that the mean dissolution time for all Taguchi experimental runs were constant and almost the cumulative percent of drug release throughout the test time was predicted to release the drug for in sustained manner for eight hours given in Table 6 . While the MDT for Run $3_{3}^{\mathrm{a}}$ and Run $4_{4}^{\mathrm{a}}$ of 0.757755 and 0.674866 suggests rapid drug release in first intervals of test period indicating substantial increase in concentration of alpha tocopherol. Alpha tocopherol is a lipophilic potential antioxidant that might interact with membrane phospholipids and modulates the membrane fluidity and hence lead to the rapid solubilization of the drug and it may be anticipated that the critical micelle concentration of alpha tocopherol may be greater than $1 \%$. Therefore the enhanced concentrations of alpha tocopherol above $1 \%$ lead to the rapid in vitro release of drug in initial hours and had no effect on in vitro dissolution behavior of drug. The $f_{1}$ and $f_{2}$ factors suggests that twelve out of sixteen runs were similar to reference with $f_{2}$ value between $50-100$ and Run $2_{2}^{\mathrm{a}}$, Run $16^{\mathrm{d}}$ are more closer to the reference with $\mathrm{f}_{2}$ of 61.19 and 80.48. The two side Paired-Sample ' $t$ ' test suggested significanr difference between reference and test runs and indicated Run $2^{\mathrm{a}}{ }_{2}$ to be more bioavailable and may result in enhanced absorption from the gastrointestinal tract as evidenced by use of D- $\alpha$-tocopheryl PEG 1000 succinate (TPGS), a derivative of vitamin $\mathrm{E}$ that has a relatively low critical micelle concentration and has been found to increase the oral absorption of drugs including vancomycin through micelle formation [11]. For hydrophobic drugs, increased wettability upon exposure to biological fluids may lead to improved dissolution thereby enhanced bioavailability that was in turn evidenced from the pharmacokinetic parameters where there was marked improvement in bioavailability 


\begin{tabular}{|c|c|c|c|c|c|c|c|}
\hline Time (h) & $\begin{array}{l}\text { Marketed } \\
\text { formulation }\end{array}$ & Run $2^{\mathrm{a}}{ }_{2}$ & Run $16^{d}{ }_{4}$ & $\Delta=$ Ref $-\operatorname{Run} 2_{2}^{\mathrm{a}}$ & $\Delta=\operatorname{Ref}-\operatorname{Run} 16^{\mathrm{d}}{ }_{4}$ & $\begin{array}{c}\mathbf{R}=\text { Reference/ Run } \\
\mathbf{2}^{\mathrm{a}}{ }_{2}\end{array}$ & $R=$ Reference/ Run $16^{d}{ }_{4}$ \\
\hline 1 & $17.6 \pm 0.21$ & $27 \pm 0.007$ & $21.2 \pm 0.08$ & $9.4 \pm 0.03$ & $3.6 \pm 0.14$ & $0.651852 \pm 0.42$ & $0.83 \pm 0.21$ \\
\hline 2 & $35.8 \pm 0.15$ & $44.3 \pm 0.15$ & $36.21 \pm 0.1$ & $8.4 \pm 0.17$ & $0.41 \pm 0.07$ & $0.809955 \pm 0.28$ & $0.98 \pm 0.14$ \\
\hline 3 & $51.6 \pm 0.21$ & $57.8 \pm 0.17$ & $52.13 \pm 0.12$ & $6.4 \pm 0.009$ & $0.53 \pm 0.28$ & $0.809955 \pm 0.14$ & $0.98 \pm 0.28$ \\
\hline 4 & $63.5 \pm 0.01$ & $69.6 \pm 0.07$ & $64.53 \pm 0.19$ & $6.1 \pm 0.02$ & $1.03 \pm 0.04$ & $0.912356 \pm 0.05$ & $0.98 \pm 0.02$ \\
\hline 5 & $74.4 \pm 0.28$ & $78.6 \pm 0.06$ & $75.32 \pm 0.28$ & $4.2 \pm 0.01$ & $0.92 \pm 0.7$ & $0.946565 \pm 0.32$ & $0.98 \pm 0.05$ \\
\hline 6 & $82.6 \pm 0.42$ & $86.7 \pm 0.03$ & $84.7 \pm 0.07$ & $4.1 \pm 0.02$ & $2.1 \pm 0.02$ & $0.95271 \pm 0.06$ & $0.97 \pm 0.04$ \\
\hline 7 & $91.0 \pm 0.17$ & $92.4 \pm 0.51$ & $94.47 \pm 0.12$ & $1.4 \pm 0.35$ & $3.47 \pm 0.17$ & $0.984848 \pm 0.12$ & $0.96 \pm 0.01$ \\
\hline \multirow[t]{2}{*}{8} & $95.5 \pm 0.01$ & $97.7 \pm 0.007$ & $98.42 \pm 0.08$ & $1.2 \pm 0.06$ & $2.92 \pm 0.08$ & $0.98759 \pm 0.10$ & $0.97 \pm 0.007$ \\
\hline & & & & & & ${ }^{*} t=5.2006$ & ${ }^{*} \mathrm{t}=4.281$ \\
\hline
\end{tabular}

${ }^{*} t$ indicates the significant difference at $5 \%$ level

Table 7: Test for Comparison of means of reference and optimized Taguchi experimental runs using a two side Paired-Sample ' $t$ ' test ( $\mathrm{n}=6$ ).

rate and extent compared to the pure drug. Significant differences were found in $\mathrm{AUC}_{0-24} \mathrm{~h}$ as well as in $\mathrm{C}_{\max }$ and $\mathrm{t}_{1 / 2}$ between the test and reference formulations as shown in the Table 6 . The hypothesized mechanism is the interaction and fluidization of membrane phospholipids thereby assisting in drug transport by enhancing the lipid solubility [12] as in the case of 1-deamino-8-D-arginine-vasopressin where the transport of lysophosphatidyl choline treatment increased the apparent permeability coefficient, $\mathrm{P}_{\text {app }}$ even under circumstances where the cell monolayer integrity was only slightly altered and also evidenced by 8 fold increase in concentration of miconazole indicating that the enhancement effect involved high partition of miconazole into the skin by the use of phospholipid and dodecyl 2-(N,N-dimethyl amino) propionate $[13,14]$. Therefore phosphatidylcholine embedded micellar systems are responsible for enhanced permeability. The difference in three types of phospholipids may be owed to the difference in percentage of phosphatidyl choline (PC) as evidenced by the solubility of miconazole that increased in proportion to the hydrogenated phosphatidylcholine concentration [14]. Run with Phospholipon 90H containing 90\% PC and $4 \%$ of lyso phosphatidyl choline, the byproduct of PC resulted in better in vitro dissolution and higher drug release. These results are similar to that of bile salts containing soybean phosphatidylcholine and sodiumdeoxycholate which were used as absorption enhancers for bioavailability of fenofibrate that showed 1.57 fold in bioavailability compared to liposomes containing soybean phosphatidylcholine and cholesterol [15]. Hence use of alpha tocopherol and phospholipids at lower concentrations and may be potential and safe solubilizers for improving dissolution thereby absorption of poorly water soluble drugs similar to dendrimers [16]. These may also have potential effects on other poorly water soluble drugs like griseofulvin, cefotaxime sodium, ceftazidime pentahydrate, cyclosporin A and lovastatin that made use of alpha tocopherol and medium chain fatty acids (sodium caprylate, caprate), cyclodextrins ( $\beta$-cyclodextrin, hydroxypropyl $\beta$-cyclodextrin) and bile salts (sodium cholate, deoxycholate) for solubilization of drug and absorption enhancement [17]. There may be effect on absorption kinetics similar to norfloxacin where the absorption kinetics was markedly accelerated when disodium ethylenediaminetetraacetate (EDTA) and sodium caprate were used [18]. In conclusion, use of solubilizers may be a suitable way to improve the bioavailability of drugs with low water solubility.

\section{Conclusions}

The developed formulations were found to have positive impact on in vitro dissolution behavior of highly lipophilic and poorly water soluble fenofibrate compared to the marketed formulation. Alpha tocopherol was found to be effective at $1 \%$, while Phospholipon $90 \mathrm{H}$ was found to be effective at $2 \%$. The hypothesized mechanism is the interaction and fluidization of membrane phospholipids. Hence, formulating sustained release fenofibrate pellets with alpha tocopherol and phospholipids using fluid bed coating technique was found to be more reliable, to improve the oral bioavailability of poorly water soluble and highly lipophilic drugs like fenofibrate and the like.

\section{Acknowledgements}

The authors are grateful to R.A. Chem Pharma, India for extending their help to work in Formulation Research and Development and carryout the scientific work. The authors also wish to thank Lipoid $\mathrm{GmbH}$, USA for providing gift samples of Phospholipon $80 \mathrm{H}$ and Phospholipon $90 \mathrm{H}$.

\section{References}

1. Francis MF, Piredda M, Winnik FM (2003) Solubilization of poorly water soluble drugs in micelles of hydrophobically modified hydroxypropylcellulose copolymers. J Control Release 93: 59-68.

2. Hauss DJ (2007) Oral lipid-based formulations. Adv Drug Deliv Rev 59: 667676.

3. Keating GM, Ormrod D (2002) Micronised fenofibrate: an updated review of its clinical efficacy in the management of dyslipidaemia. Drugs 62: 1909-1944.

4. Guay DR (2002) Update on fenofibrate. Cardiovasc Drug Rev 20: 281-302.

5. Fruchart JC, Sauzieres J, Clavey V, Plancke MO (1989) Antioxidant therapy and uptake of human oxidized LDL by macrophages. Ann NY Acad Sci 570 447-448.

6. Nielsen PB, Mullertz A, Norling T, Kristensen HG (2001) The effect of a-tocopherol on the in vitro solubilization of lipophilic drugs. Int J Pharm 222 217-224.

7. Balfour JA, McTavish D, Heel RC (1990) Fenofibrate. A review of its pharmacodynamic and pharmacokinetic properties and therapeutic use in dyslipidaemia. Drugs 40: 260-290.

8. Pund S, Joshi A, Vasu K, Nivsarkar M, Shishoo C (2010) Multivariate optimization of formulation and process variables influencing physicomechanical characteristics of site-specific release isoniazid pellets. Int J Pharm 388: $64-72$.

9. Rahman MA, Ahuja A, Baboota S, Bhavna, Bali V, et al. (2009) Recent Advances in Pelletization Technique for Oral Drug Delivery: A Review. Curr Drug Deliv 6: 122-129

10. Bolton S, Bon C (2004) Statistical inference: Estimation and hypothesis testing. In: Swarbrick J, editor. Pharmaceutical statistics: Practical and clinical applications. Newyork: Marcel Dekker 96-150.

11. Prasad YV, Puthli SP, Eaimtrakarn S, Ishida M, Yoshikawa Y, et al. (2003) Enhanced intestinal absorption of vancomycin with Labrasol and D-alphatocopheryl PEG 1000 succinate in rats. Int J Pharm 250: 181-190.

12. Kirjavainen M, Mönkkönen J, Saukkosaari M, Valjakka-Koskela R, Kiesvaara J 
Citation: Indira TK, Lakshmi PK, Balasubramaniam J , Rajesh YV (2012) Enhancement of Bioavailability of Fenofibrate with Alpha Tocopherol and Phospholipids as Solubilizers. J Bioequiv Availab S14. doi:10.4172/jbb.S14-006

Page 8 of 8

et al. (1999) Phospholipids affect stratum corneum lipid bilayer fluidity and drug partitioning into the bilayers. J Control Release 58: 207-214.

13. Hovgaard L, Brondsted H, Nielsen HM (1995) Drug delivery studies in Caco-2 monolayers. II. Absorption enhancer effects of lysophosphatidylcholines. Int J Pharm 114: 141-149.

14. Fujii M, Büyüktimkin S, Büyüktimkin N, Rytting JH (2002) Enhancement of skin permeation of miconazole by phospholipid and dodecyl 2-(N,N-dimethyl amino) propionate (DDAIP). Int J Pharm 234: 121-128.

15. Chen Y, Lu Y, Chen J, Lai J, Sun J, et al. (2009) Enhanced bioavailability of the poorly water-soluble drug fenofibrate by using liposomes containing a bile salt. Int J Pharm 376: 153-160.

16. Lin Y, Fujimori T, Kawaguchi N, Tsujimoto Y, Nishimi M, et al. (2011)
Polyamidoamine dendrimers as novel potential absorption enhancers for improving the small intestinal absorption of poorly absorbable drugs in rats. J Control Release 149: 21-28.

17. Sharma P, Varma MV, Chawla HP, Panchagnula R (2005) Absorption enhancement, mechanistic and toxicity studies of medium chain fatty acids, cyclodextrins and bile salts as peroral absorption enhancers. Farmaco 60: 884893.

18. Dos Santos I, Fawaz F, Lagueny AM, Bonini F (2003) Improvement of norfloxacin oral bioavailability by EDTA and sodium caprate. Int J Pharm 260: $1-4$. 